\title{
How lateral inhibition and fast retinogeniculo-cortical oscillations create vision: A new hypothesis
}

\author{
Ravinder Jerath MD ${ }^{\mathrm{a}, *}$, Shannon M. Cearley BS ${ }^{\mathrm{a}}$, Vernon A. Barnes PhD ${ }^{\mathrm{b}}$, Elizabeth Nixon-Shapiro MSMI ${ }^{\mathrm{c}}$ \\ a Augusta Women's Center, Augusta, GA, USA \\ ${ }^{\mathrm{b}}$ Augusta University, Augusta, GA, USA \\ ${ }^{\mathrm{c}}$ Nixon Medical Media, Rome, GA, USA
}

\section{A R T I C L E I N F O}

\section{Article history:}

Received 1 April 2016

Accepted 21 September 2016

\section{Keywords:}

After-image

Human vision

Lateral inhibition

Phototransduction

Physiological oscillations

\begin{abstract}
A B S T R A C T
The role of the physiological processes involved in human vision escapes clarification in current literature. Many unanswered questions about vision include: 1) whether there is more to lateral inhibition than previously proposed, 2) the role of the discs in rods and cones, 3 ) how inverted images on the retina are converted to erect images for visual perception, 4) what portion of the image formed on the retina is actually processed in the brain, 5) the reason we have an after-image with antagonistic colors, and 6) how we remember space. This theoretical article attempts to clarify some of the physiological processes involved with human vision. The global integration of visual information is conceptual; therefore, we include illustrations to present our theory. Universally, the eyeball is $2.4 \mathrm{~cm}$ and works together with membrane potential, correspondingly representing the retinal layers, photoreceptors, and cortex. Images formed within the photoreceptors must first be converted into chemical signals on the photoreceptors' individual discs and the signals at each disc are transduced from light photons into electrical signals. We contend that the discs code the electrical signals into accurate distances and are shown in our figures. The pre-existing oscillations among the various cortices including the striate and parietal cortex, and the retina work in unison to create an infrastructure of visual space that functionally "places" the objects within this "neural" space. The horizontal layers integrate all discs accurately to create a retina that is pre-coded for distance. Our theory suggests image inversion never takes place on the retina, but rather images fall onto the retina as compressed and coiled, then amplified through lateral inhibition through intensification and amplification on the OFF-center cones. The intensified and amplified images are decompressed and expanded in the brain, which become the images we perceive as external vision. Summary: This is a theoretical article presenting a novel hypothesis about the physiological processes in vision, and expounds upon the visual aspect of two of our previously published articles, "A unified 3D default space consciousness model combining neurological and physiological processes that underlie conscious experience", and "Functional representation of vision within the mind: A visual consciousness model based in 3D default space." Currently, neuroscience teaches that visual images are initially inverted on the retina, processed in the brain, and then conscious perception of vision happens in the visual cortex. Here, we propose that inversion of visual images never takes place because images enter the retina as coiled and compressed graded potentials that are intensified and amplified in OFF-center photoreceptors. Once they reach the brain, they are decompressed and expanded to the original size of the image, which is perceived by the brain as the external image.

We adduce that pre-existing oscillations (alpha, beta, and gamma) among the various cortices in the brain (including the striate and parietal cortex) and the retina, work together in unison to create an infrastructure of visual space that functionally "places" the objects within a "neural" space. These fast oscillations "bring" the faculties of the cortical activity to the retina, creating the infrastructure of the space within the eye where visual information can be immediately recognized by the brain. By this we mean that the visual (striate) cortex synchronizes the information with the photoreceptors in the retina, and the brain instantaneously receives the already processed visual image, thereby relinquishing the eye from being required to send the information to the brain to be interpreted before it can rise to consciousness.
\end{abstract}

\footnotetext{
* Corresponding author at: 2100 Central Avenue, \#7, Augusta, GA 30904, USA.

E-mail address: rj605r@aol.com (R. Jerath).
} 
The visual system is a heavily studied area of neuroscience yet very little is known about how vision occurs. We believe that our novel hypothesis provides new insights into how vision becomes part of consciousness, helps to reconcile various previously proposed models, and further elucidates current questions in vision based on our unified 3D default space model. Illustrations are provided to aid in explaining our theory.

(C) 2016 Augusta Women's Center. Published by Elsevier Ltd. This is an open access article under the CC BYNC-ND license (http://creativecommons.org/licenses/by-nc-nd/4.0/).

\section{Introduction to the retina}

The retina is the area in the back of the eye that contains light sensitive neurons, and is the first stage of processing visual information and sending it to the brain [5-7]. Within the retina are five types of neurons: photoreceptors, bipolar cells, ganglion cells, horizontal cells, and amacrine cells [5]. Graded electrical activity is converted into action potentials in the retina, and is transmitted to the optic nerve. Since there is a relatively short distance for information to travel within the retina, action potentials are not needed, and instead, graded potentials mediate the information the retina processes [5].

In the retina there are approximately 100 million rods, 5 million cones, and only 1 million ganglion cells [8]. The horizontal and amacrine cells are responsible for the lateral interactions, which assist the visual system in detecting contrast in intensity of light $[5,9,10]$. The human retina is inverted to require light from projected images to pass through layers of tissue to reach the photoreceptors [11]. These layers of tissue contain various cells which cause light scattering [12] (Fig. 1). Muller cells are funnel-shaped glial cells in the retina that are essential for the transmission of light due to their unique shape, orientation, and refractive index [13]. These cells act as conduits that enable light to reach the photoreceptors with minimal scattering [13], and in mammals, every Muller cell is generally coupled with a partner cone cell [14].

The photoreceptors are rods and cones, and the infoldings in cones contain the pigment that makes up nearly $80 \%$ of the total disc protein, trapping photons as they travel through the outer segments [15]. Rods are highly sensitive to light, insomuch that only seven are needed to detect a single photon of light in a dark environment [15], whereas, cones are less sensitive and cannot detect light if photons are fewer than 100 [5]. The fovea consists of a concentrated amount of elongated cones for color vision acuity in bright light (which communicate with midget bipolar and midget ganglion cells), and extra-foveal regions for acuity in dimmer light, yet no rods exist within this area [8].
Ganglion cells synchronize the internal circadian rhythm with the external light-dark cycle, without attaching to the individual photoreceptors because they are reserved for image processing [16]. Our theory proposes that retinal layers and cells: 1) code distances, 2) compress the information about the images and distances, and 3) integrate the information with the striate cortex. Since the retina is considered to be part of the brain, we consider it as a smaller, compressed space that projects information into the larger neural space within our brains, bringing the brain to the eye. The retina is also the place where images are coiled and compressed as right side up images before being sent to the brain to be decompressed and expanded, according to our theory.

Images we see are processed in the retina [17], through biochemical cycles, resulting in amplification and intensity of images and their color contrasts [17]. When light enters our eyes through the cornea, it is focused in the retina on the rods and cones causing the initial image-which we do not see-to fall on the ON-center cells [5]. Photoreceptors synapse with horizontal cells and bipolar cells forming a triad, inhibiting the surrounding receptor cells, enhancing contrast, and hyperpolarizing or depolarizing bipolar cells [18]. The chain of interactions that takes place through parallel processing of visual information starts with input received from retinal photoreceptors sent via their interconnecting bipolar cells to reach the ganglion cells. Glutamate release at ribbon-type synapses allows for the transfer of information by photoreceptors and bipolar cells $[19,20]$.

In the vertebrate retina, glutamate is not only the major neurotransmitter [21], it is also the used by the first visual synapse [22]. Glutamate depolarizes OFF-center ganglion cells and hyperpolarizes ON-center ganglion cells because of their ionotropic receptors. Reduction in glutamate has an opposite effect-hyperpolarizing OFF-center cells, and depolarizing ON-center cells [5]. ON-center ganglion cells respond to light by producing a burst of electrical activity, but the response to light in OFF-center ganglion cells results in decreased electrical activity. The cells respond with a burst of action potentials when the light is turned off [5] due to

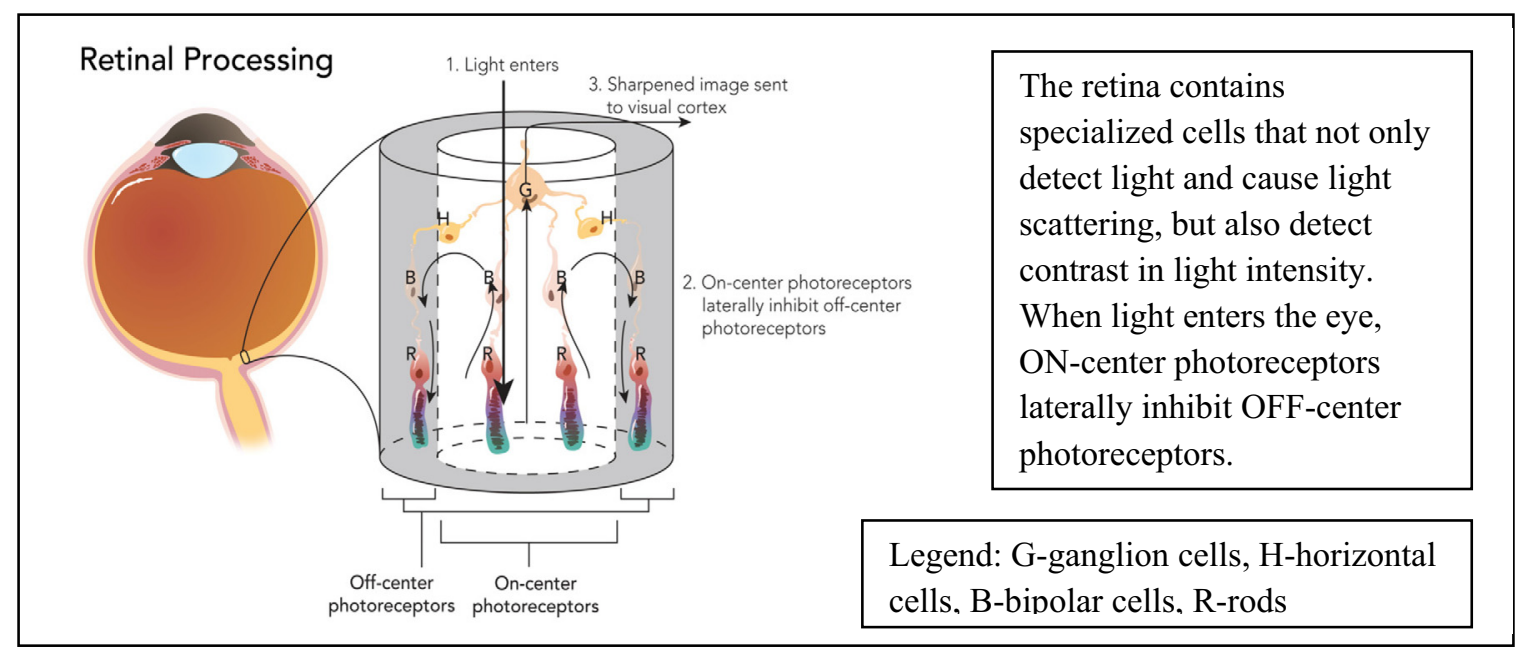

Fig. 1. Retinal Processing 
a high rate of glutamate release in the dark, which causes postsynaptic receptors to be activated [22]. Since bipolar cells also have graded potentials, the gradual decrease in light causes hyperpolarization of ON-center cells, and depolarization of OFF-center cells [5], regulating synaptic input from photoreceptors to bipolar and horizontal cells [22].

In the retina, two types of amacrine cells are fundamental in processing scotopic visual signals: AII amacrine cells and A17 amacrine cells. ON- and OFF-cone bipolar cells receive feedback signals from AII amacrine cells, while rod bipolar cells receive the GABAergic inhibition from A17 amacrine cells [23]. Rod bipolar cells are solely ON-type bipolar cells (there are no OFF-type rod bipolar cells), and there is no direct synaptic contact between rod bipolar cells and ganglion cells. In order for rod bipolar cells to communicate with ON- and OFF-ganglion cells, they must use AII amacrine cells $[24,25]$. Amacrine interneurons are highly diversified and are essential for processing visual information in the retina [26]. Amacrine cells not only release glutamate signals received by the special receptors (eye-enriched kainate receptors) in photoreceptors, but their feedback regulation is responsible for maintaining light sensitivity in ambient light [27].

The substrate that aids the retina in visual processing is found in cone bipolar cells. Small stimuli is encoded by ON-cone bipolar cells with axons branching into the central inner plexiform layer, while both small and large stimuli are encoded by ON-cone bipolar cells with axons branching into the proximal inner plexiform layer near the ganglion cell layer. Parallel processing of color and luminance make this possible. Furthermore, the spatial encoding properties of the ON-cone bipolar cells is determined by information received from amacrine cells [7]. AII amacrine cells are fundamental in the primary rod pathway because rod bipolar cells use AII amacrine cells to send information to ON- and OFF-cone bipolar cells $[24,25]$. When rod bipolar cells excite AII amacrine cells, excitatory information is sent to ON-cone bipolar cells, inhibiting OFFcone bipolar cells, showing how the ON and OFF pathways mirror one another [24].

We propose that the horizontal cells in the outer plexiform layer, and possibly the amacrine cells in the inner plexiform layer, are powered by the striate cortex, parietal cortex, and various association cortices via ganglion cell layers, through alpha, beta, and gamma oscillations. We suggest that the entire basis of these highly active neurons is to carry the precise information and visuo-spatial information (especially around the cones in the fovea) to the rods.

\section{Phototransduction}

Phototransduction is the conversion of photons into neural signals that the brain can understand [28], and takes place in the retina [17]. Phototransduction converts light to electro-chemical signals at each disc through utilization of rhodopsin, along with other proteins and transmitters resulting in vision [29]. The first step in visual transduction is the phototransduction cascade which converts light into chemical signals [30]. Photochemicals adjust light intensities to greater than 10 logarithmic units [31]. Photoreceptors also act as "photomultipliers with gains of $10^{5}(100,000)$ times" [18]. The second step converts the chemical image into an electrical image through graded electrical potentials [32], which we hypothesize is responsible for the coiled and compressed images that are intensified and amplified in the OFF-center cones in the retina before the image is sent to the brain as a decompressed and expanded final image.

This chemical-to-electrical conversion of information takes place via cyclic-nucleotide-gated (CNG) channels. The levels of cyclic guanosine monophosphate (cGMP) in the cytoplasm closes CNG channels, causing the membrane potential to hyperpolarize the cell from $-40 \mathrm{mV}$ to $-70 \mathrm{mV}$, blocking glutamate release [31]. The electric chemicals extend through the photoreceptors and visual system [33], hyperpolarizing them by biochemically changing 11-cis retinal to all-trans retinal in rhodopsin. cGMP-gated channels close [34], resulting in the biochemical change of all-trans retinal into 11-cis retinal. This biochemical change regenerates rhodopsin [34] through the retinoid visual cycle [29], returning the cell to a dark state [34].

Rhodopsin is fundamental in vision formation. Not only does it cover more than half of the surface of rod discs, rod cells need it in order to survive [29]. Once photons enter the photoreceptors, rhodopsin absorbs them, and they become photoisomerized and changed from the 11-cis to the all-trans configuration, all of which takes place in $10^{-12} \mathrm{~s}$ [15]. We interpret visual processing as instantaneous because we are incapable of detecting a time interval less than $40 \mathrm{~ms}$. Any two events that are separated by less than $40 \mathrm{~ms}$ appear as happening simultaneously [35], suggesting that there is a lag time in which processing must take place before one can perceive anything externally. This supports our theory in that as these biochemical processes take place, the images are projected directly into the brain through pre-existing oscillations that allow the retina and brain to communicate in real-time, because this neural network was formed in utero [36-39].

Cone photoreceptors need to absorb several photons simultaneously to reach the detection threshold in the dark current's outer segment because photon photocurrents have small amplitudes [40]. Dark currents are also known as dark noise resulting from the constant changes in cGMP and free, unbound calcium concentrations originating in darkness from synthesis (from guanylate cyclase) and hydrolysis (from phosphodiesterase) of cGMP [40]. This causes rods to be more sensitive to light, and causes cone photocurrents to be noisier, have smaller peak amplitudes, and travel faster than rods. Smaller peak amplitudes and faster travel causes cones to be less sensitive to light than rods [40]. This suggests that photoreceptors generate the dark current from photopigments in the discs of the photoreceptors. Since dark currents exist without illumination and increase when there is light, it is possible that this is the basis for the formation of the pre-existing oscillations that play a fundamental role in vision.

\section{Lateral inhibition}

Vision is understood to primarily form in the retina, with final visual perception occurring in the brain [41], and lateral inhibition is considered to be the first stage in vision processing. Lateral inhibition is a phenomenon observed in many sensory organs including the retina, but its retinal function is currently explained as being responsible for the demarcation of edges in shades of dark and light colors [42] in OFF-center cones or ON-center cones, depending upon the type of ganglion cells and receptive field [41]. Lateral inhibition is also recognized as being responsible for removing the visual redundancy in natural images and reducing visual blurs. Lateral inhibition works together with horizontal and bipolar cells to provide information to distant rods and cones, helping to enhance edges and contours for recognition of contrast in visual images [43]. This explanation may be true; however, it does not clarify other physiological and clinical observations such as 1) erection of vision despite the initial image inversion, 2) purpose of stacked discs in individual photoreceptors, 3) why negative after-images have opposite colors, 4) why photoreceptors, bipolar cells, and ganglion cells are anatomically vertical, and 5) how vision occurs vividly and coincides with binocular threedimensional vision. 
We theorize that the horizontal layers integrate all discs accurately to create a retina that is pre-coded for distance, and that lateral inhibition is not only responsible for demarcation of edges, but also for the reversed colors we see in afterimages. It is possible that the ON-center rods and cones are "electrically" lit up and provide a margin contrast enhancement as seen in lateral inhibition [44].

\section{Visual circuits}

Visual circuits in reptilian, avian, and mammalian species develop independently from sensory experience, and once the eyes open, visual development continues and becomes further refined [45]. Origin and nature of activity of retinal waves lacks in vivo testing in humans, but is at the seat of what drives the controversy of how retinal waves contribute to the development of neural circuitry. Prior hypotheses suggest they could play a part in forming maps for "orientation, direction, and ocular dominance" prior to vision [37], while experimental data has indicated that retinal waves develop early on, are critical in the developing visual system interconnections, and help prepare the circuitry required for visuomotor learning and behavior [45]. In humans, spontaneous retinal activity is generated by retinal neurons before vision takes place, creating functional maps in higher visual areas [37,38,46]. The supposition is that retinal development and subsequent retinal waves link together the lateral geniculate body and cortex, thereby being a major contributor in the formation of an "internal" neural space which forms the infrastructure of visual consciousness.

Another aspect of visual consciousness lies in an electric potential field in the eyes. Human eyes have an electric potential field that is constant and independent of light stimulation, and can be detected in darkness or when our eyes are closed [18]. The oculogram is based on the lower electrical potential by $1 \mathrm{mV}$ of the cornea and anterior vitreous humor, resulting in a steady and unchanging electrical field [18]. It is this uninterrupted electric potential field that is the constituent of the combined space that is formed by both eyes and merged binocularly into a visuospatial consciousness [4].

\section{Vision and brain oscillations}

Cortical magnification means that there is more cortical space and more cortical neurons devoted to processing visual information received from the fovea, than what is needed to process visual information in other areas of the retina [35]. Prior to reaching the cortex, not only are photon signals amplified, but visual information is irreversibly modified in the retina [35]. Signal amplification and photon amplification are not interchangeable terms. Signal amplification refers to "an electric current and release of glutamate," that happens when photoreceptors detect photons transducing them to chemical-neural signals through biochemical cascades [35].

In humans, visual signals have already undergone much of their processing in the retina, prior to reaching the visual cortex [47], and information received from the optic nerve is sent to the thalamus, midbrain, and cerebral cortex as a set of neural maps $[37,45]$. Oscillations within the retina, lateral geniculate nucleus (LGN), and cortex indicate synchronization between neurons and oscillatory activity in the LGN at $60-120 \mathrm{~Hz}[48,49]$. Also, dissociation of subcortical and cortical oscillations resulting from stimulus movement provides evidence for separate subcortical and cortical mechanisms $[48,49]$. These separate subcortical and cortical mechanisms result in cortical synchronization at $30-60 \mathrm{~Hz}$ dominating cortical oscillations $[48,49]$.

Alpha oscillations vibrate at $8-14 \mathrm{~Hz}[50,51]$ and strongly influence brain activity, being responsible for more efficient visual pro- cessing than other brain rhythms [50]. In the human brain, alpha oscillations are the dominant oscillations (at $10 \mathrm{~Hz}$ ) [52], and alpha rhythm is strongest when one is in a relaxed state with the eyes closed, or when a person is in a dark environment [52,53]. This is the constituent of the combined space formed by both eyes and merged binocularly into visuospatial consciousness [4]. We postulate that retinal development and subsequent retinal waves link together the lateral geniculate body and cortex, and an internal dynamic infrastructure (or neural space) is formed by various levels of membrane potential-based oscillations that link together with the pupils and photoreceptors.

Alpha oscillations are also fundamental in central nervous system functioning [54]. Cortical inhibition is evidenced by alpha power, and faster reaction times correlate with stronger alpha power [55]. Stronger desynchronization and faster resynchronization were found in people with higher working loads and in those who held more items in memory during memory experiments, while an increased stimulus-driven alpha desynchronization was found to be coupled with more effective stimulus coding [55]. Once desynchronization ends, resynchronization begins and alpha oscillations return to their original levels [55]. The supposition is that this is indicative of alpha desynchronization being affected by an increase of new information, possibly resulting from an increase in visual information stimulating a greater number of neurons.

Gamma-band oscillations vibrate at a frequency of $28-48 \mathrm{~Hz}$ [54], but those greater than $30 \mathrm{~Hz}$ are important for the neural coding during cognitive processes, and in the visual cortex they are associated with fixed patterns of eye movement [56]. Sustained visual stimulation induces gamma oscillations to be in the range of $50-70 \mathrm{~Hz}$ [57]. Synchronization of gamma oscillations are vital in: 1) cortico-cortical communication (at $30-100 \mathrm{~Hz}$ ) [51,54,58,2] neural integration needed for cognitive functioning [54,3] are closely connected with visual speed, accuracy, and discrimination, suggesting their importance in organizing and analyzing visual information [56].

The premise is that alpha and gamma rhythms involve oscillations of membrane potentials, and together they play a major role in forming an "internal" neural space that forms the infrastructure of visual consciousness that is a dynamic template reducing external space into an internal world space. We postulate that these fast oscillations 'bring' the faculties of cortical activity to the retina (Fig. 2). By this we mean that the visual (striate) cortex, which has neurons that code for color, distance, and position in space of external objects, synchronizes the information with the photoreceptors. Additionally, this fast synchronization creates a preexisting space formed within the eye where visual information can be projected. This infrastructure contains the visual image along with its corresponding colors, distances, and position in space to be immediately recognized by the brain through the fast oscillations, thereby relinquishing the eye from being required to send the information to the brain to be interpreted before we become consciously aware of it. We consider this infrastructure essential as it is needed in order to "plug in" the visual image.

\section{Our new theory of vision and brain oscillations}

This new theory of vision adduces that when light enters the eye, the image that is formed on the retina is never inverted. We explain this with a metaphor of a spring being compressed and decompressed at different stages in the visual process. To begin, an image is similar to a spring that has been stretched. As that image enters the eye, it becomes coiled and compressed. This coiling and compressing represents the graded potentials that are intensified and amplified by lateral inhibition in the OFF-center cones and decompressed and expanded in the brain to the original 


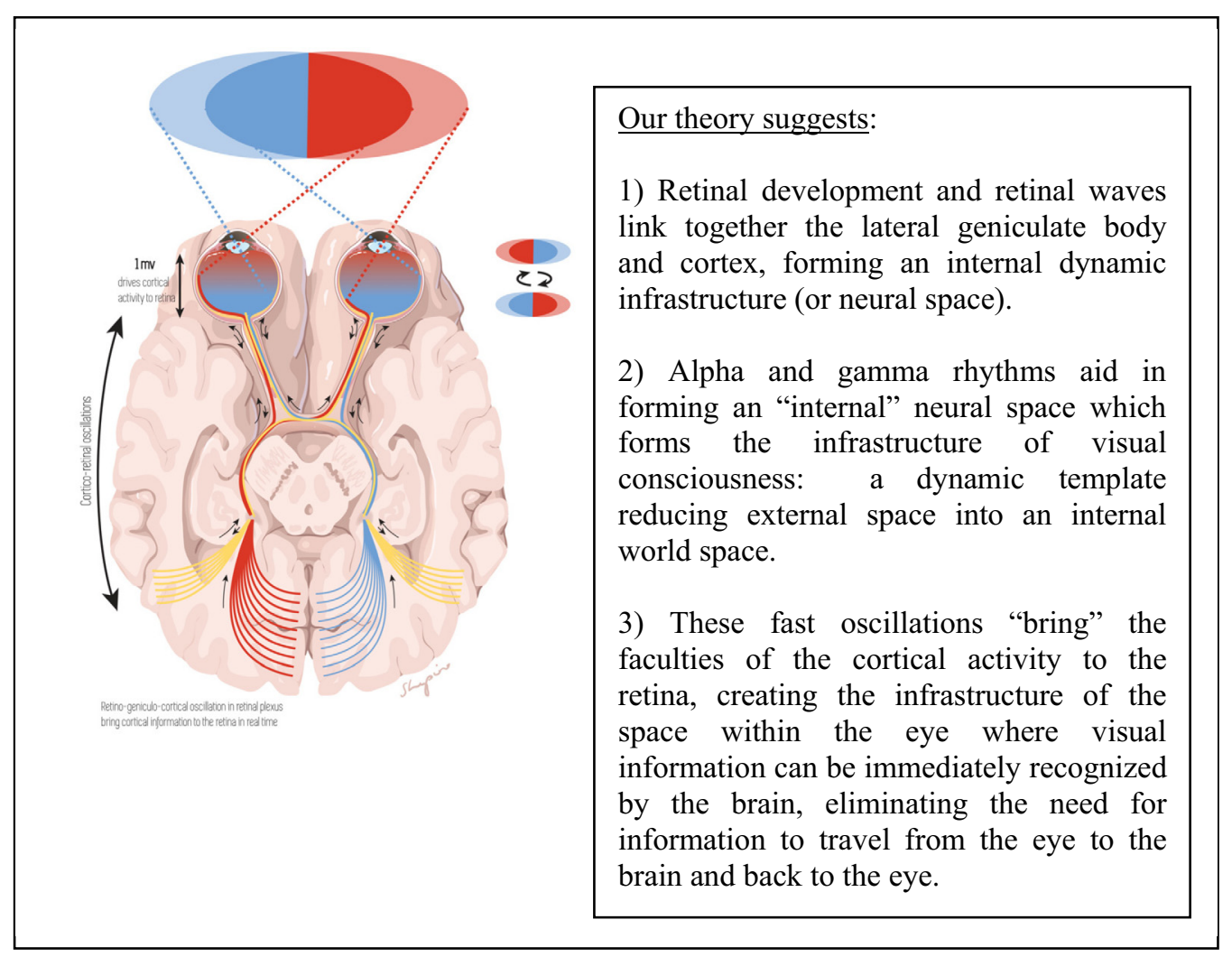

Fig. 2. Cortical Activities of the Brain.

image. This expanded image is perceived as the external image by the brain. The brain never receives an inverted image so there is no need for the image to be processed right side up as is currently understood, meaning that the image that falls on the retina is already right side up prior to entering the brain. The brain receives the image correctly alleviating the need to process the image then return it to the retina. This is possible because the brain projections from the retina are oscillating at $8-14 \mathrm{~Hz}$ with visual and association cortices [50,51], and the oscillating activity "brings" the brain faculties to the retina within milliseconds. Visual information can reach the cortex through the retinogeniculo-cortical pathway [59], and the rich network of neurons via horizontal cells, amacrine cells, and inner and external plexuses [60,61], which happens through the fast back-and-forth oscillations $[48,49]$. This is what we have named the pre-existing infrastructure of the visual consciousness space.

It is possible that each photoreceptor acts as an individual eye with tunnel vision that converts light to graded potentials (Fig. 3). All the tiny images combine in the OFF-center cones and combine to form the final image we perceive as vision. Furthermore, all of the photoreceptors are integrated with the brain by the retinal plexus (inner and outer layers that are oscillating with the brain) to form a cohesive image we perceive as an image external to us.

Our theory conjectures that millions of ON-center cones receive the initial image, process it through phototransduction, and carry opposite colors, which is evidenced by the universal phenomenon seen during a color afterimage [62,63]. This information is processed almost instantly and exclusively, in the retina via horizontal cells (powered by ongoing cortical synchronization), with an almost equal number of OFF-center photoreceptors with manyfold magnification and intensification. This forms the final image that we see and perceive as the external world. The millions of OFF-center cones and rods project this final image to the brain, inserting it into the visual fields we see as the external image/world. Fig. 3 illustrates that the margin enhancements as ONcenter are shown darker than the OFF-center photoreceptors after lateral inhibition.

\section{After-images}

After-images are a visual phenomenon in which a faint image continues to appear after exposure to the image has ceased, due to residual images from ON-center photoreceptors that are not yet processed, showing faint colors that have been reversed [2]. They are understood to occur as a result from neural adaptation, but since neural adaptation in the visual system happens so frequently, the exact neural location remains unknown [63]. Afterimages can be in the form of positive after-images, which match the original image, or negative after-images which have reverse coloring of the original image. Though recent studies have shown some cortical involvement in both positive [64,65] and negative after-images [66], it has long been maintained that they are the result of overstimulation and desensitization of photoreceptors [2]. After-images cannot simply be explained as photoreceptor desensitization or pigment bleaching because light can occur at mid-photopic levels, and postreceptor rebound responses in retinal ganglion cells create an after-image signal when lights are below the photoreceptor bleaching level [63]. It is possible that we may be seeing the memory of figures and colors, substituting those into the real image.

In primates, it has been shown that after-images 1) are generated in the retina, 2) can be modified by cortical processes, and 3 ) ganglion cells generate rebound images post stimulus exposure [63]. We suggest that this is preliminary evidence supporting our theory that the images are initially erect because after-images are erect. The theory that images are not inverted on the retina 


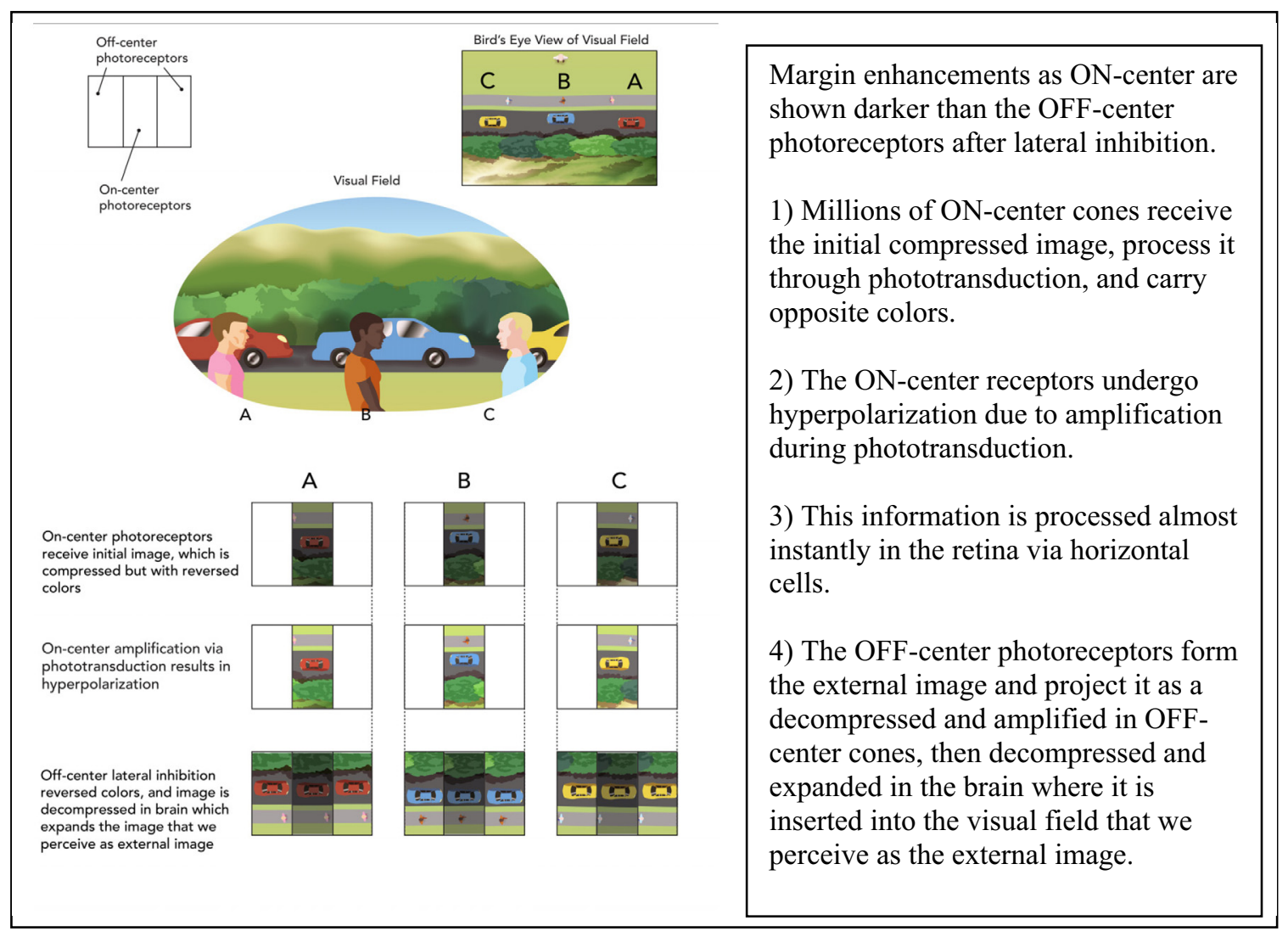

Fig. 3. Photoreceptors.

at any point in vision, but rather coiled and compressed on the retina, then intensified and amplified in OFF-center photoreceptors then are decompressed and expanded in the brain to the original size of the image is conveyed. We do not deny that cortical processing is involved in the phenomenon of after-images, but it is likely much less involved than the high thalamocortical involvement during normal vision.

The speculation is that after-images happen on the retina resulting from the electro-chemical image during the process of visual perception on the ON-center photoreceptors (Fig. 4). This means that the final image we see is not the initial ON-center image, but rather the OFF-center collective image. This further extends the idea that the after-image we see is the initial electro-chemical image, which stays longer than the electrical graded potential image. This is the initial electrochemical image we see when 1) we close our eyes after looking at an image, or 2) look at a blank canvas after staring at an optical illusion.

\section{Current accepted theory of vision}

Currently in neuroscience, conscious perception of visual information is considered to occur in the primary visual cortex, and current theory on vision suggests that electrical signals coding the visual image are transmitted to the visual cortex for processing [35], (Fig. 5-Flowchart 1). However current explanation of visual processing does not factor in the temporal aspects of the fast synchronization of visual and association cortex to retina at alpha 8$14 \mathrm{~Hz}[50,51]$, beta at $15-30 \mathrm{~Hz}$ [51] and gamma at $30-100 \mathrm{~Hz}$ $[51,54,58]$, or the lesser understood high-gamma activity between 60 and $200 \mathrm{~Hz}$ [67]. This may lead to the conclusion that detailed, moving 3D visual scenes cannot be transmitted in the vivid details from 100 million rods and 5 million cones via only 1 million gan- glions and replayed on visual and association cortices where it can be visualized in detail that we see along with color conversion and up-right image formation. Moreover the real time accurate discernment of motion would be even more problematic.

\section{A new theory of vision}

Retinogeniculo-cortical oscillations form a pre-existing visual space that we experience when our eyes are closed because: 1) these pathways synchronize when we close our eyes [1,2] closing the eyes can affect the interoceptive resting state that is responsible for imagination and multisensory activity [3], (Fig. 6-Flowchart 2). The synchronization of retinogeniculo-cortical pathways serves to create an internal grid that accurately codes for distance generated with discs from the photoreceptors [4]. The initial light and image induce electro-chemical changes at the discs when transferred to ON-center bipolar cells, stimulating the ganglion cells in graded electrical potentials. These potentials are transferred back to the photoreceptors via lateral inhibition along with associated synchronized input from the thalamus and cortex. Moreover, the graded potentials are the electrical encoding of the original image, and the rods and cones serve as receptors for the final version of the image we perceive.

The visual space within the eyes is formed by the retinogeniculo-cortical oscillations, and the images are merged into a single fovea which is attached to a ganglion layer $[4,68]$. These retinogeniculo-cortical oscillations provide continuous communication between the retina and the brain, thereby forming a seamless view of our perception of external vision. This new theory of vision explains that an image is not formed on the retina inverted, but rather it is coiled, compressed graded potentials. The image is intensified and amplified in OFF-center photorecep- 


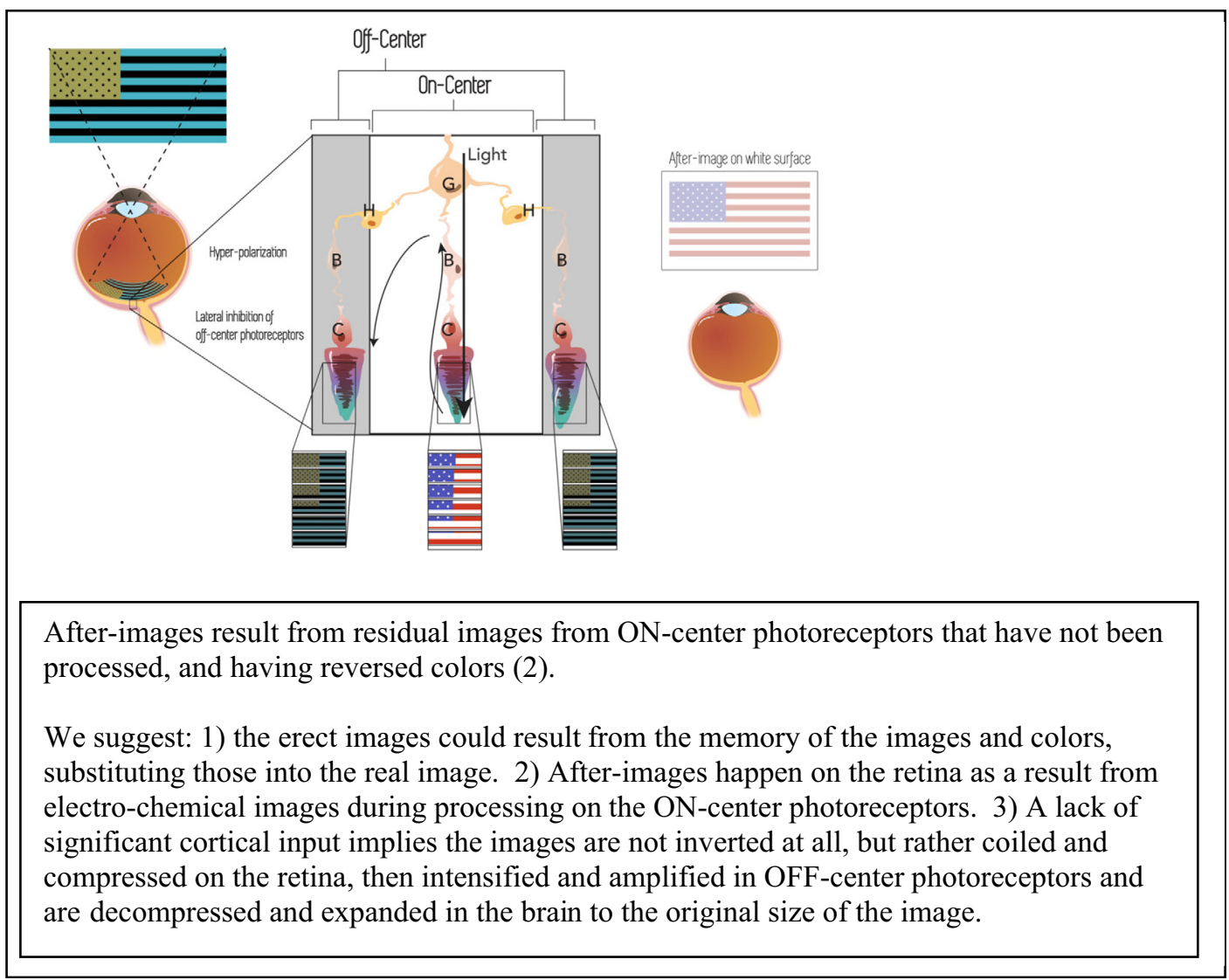

Fig. 4. After-image.

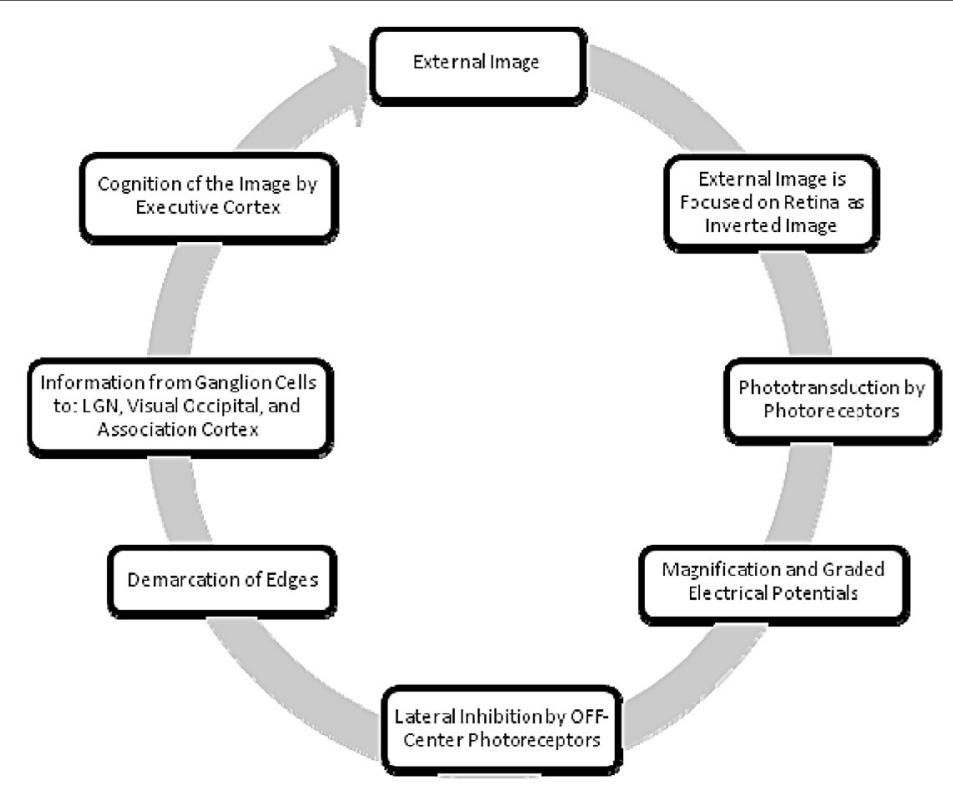

Current theory in neuroscience is that conscious perception of visual information occurs in the primary visual cortex, and that electrical signals coding the visual image are transmitted to the visual cortex for processing before vision occurs.

Fig. 5. Flow Chart 1-Current Accept Theory of Vision. 


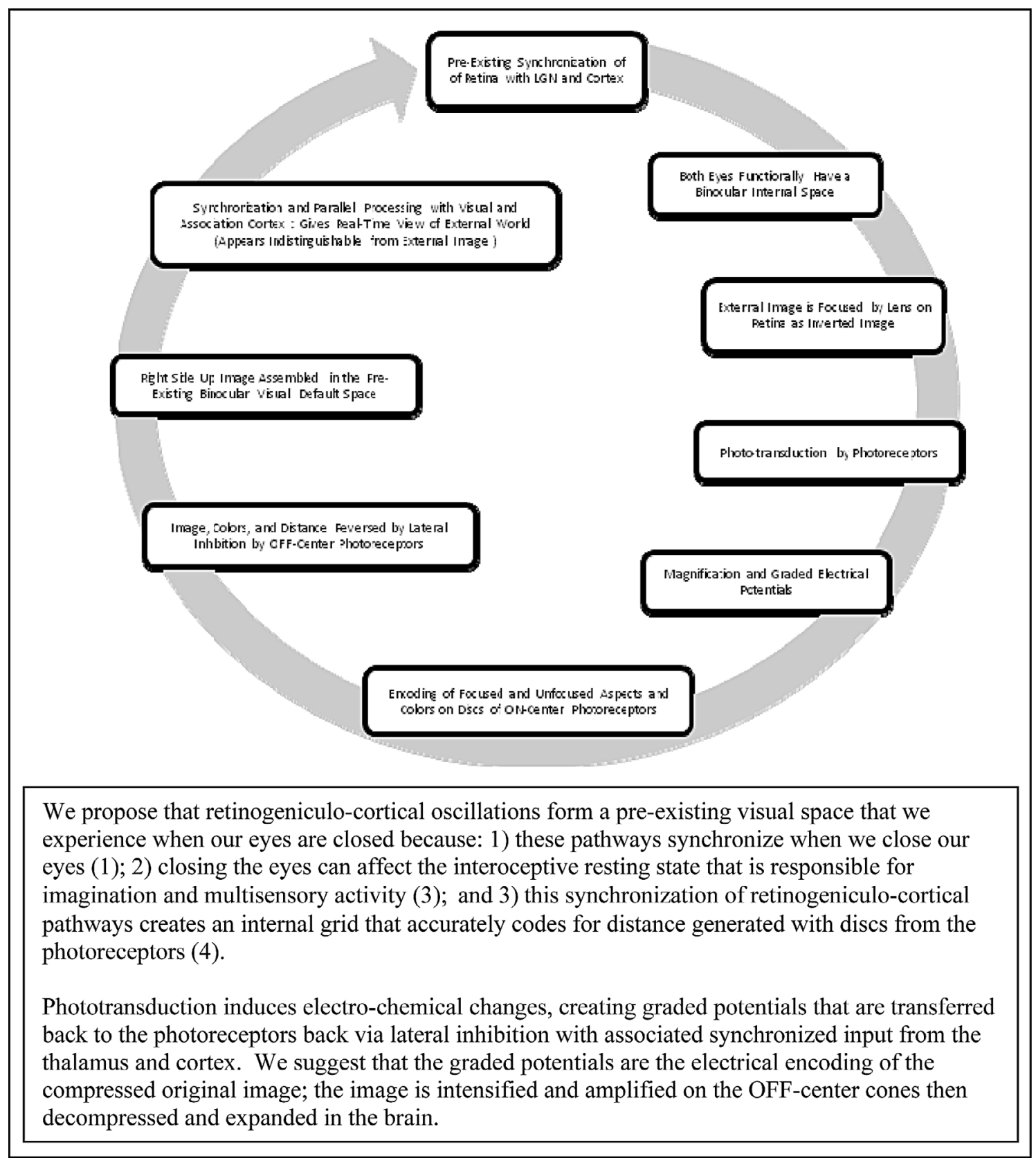

Fig. 6. Flow Chart 2-Our New Theory of Vision.

tors and are decompressed and expanded in the brain to the original size of the image, and possibly back to the ON-center cones again. The brain percepts this final image as the external image we see.

Additionally, the thalamus works as a tiny brain, tapping into the tremendous powers of the cortex within a highly active unified space that houses cognition and intrapersonal space. The lateral geniculate nucleus is known as the visual thalamus, and is innervated by relay cells that receive information and supply feedback inhibition to the visual part of the thalamic reticular nucleus. This process influences all information the primary visual cortex receives from the retina [69].

Light enters the eyes and the image falls onto the ON-center cones, coding the image, colors, and distances, and mapping them in reverse order on the individual discs. After chemical processing via rhodopsin on the discs, the electro-chemical information about the images is sent to the bipolar cells as graded potentials-graded release of glutamate-causing graded hyperpolarization in the OFFcenter bipolar cells. Since ON-center photoreceptors become hyperpolarized and generate graded potentials transmitted via ON-center bipolar cells, these potentials mirror those signals onto horizontal cells first, then onto the OFF-center photoreceptors. The final panoramic image we perceive is made possible by millions of OFF-center photoreceptors. The initial image falls on the ON-center rods or cones, is converted to chemical signals, then graded electrical signals. These graded electrical signals undergo an antagonistic process when they strike the horizontal cells and the adjoining OFF-center bipolar cells [9].

If ON-center and OFF-center cones are equal, then 3 million ONcenter cones are receiving the initial image and 3 million OFFcenter cones are reprojecting the image onto the OFF-center cones, then the same idea would apply to ON-center and OFF-center rods 
splitting them equally at 60 million each. This leads one to surmise that millions of ON-center cones receive the initial inverted image, process it through phototransduction, and carry opposite colors, which is evidenced by the universal phenomenon seen during a color afterimage [62,63]. This information is processed almost instantly and exclusively, in the retina via horizontal cells (powered by ongoing cortical synchronization), with an almost equal number of OFF-center photoreceptors with many-fold magnification and intensification [18]. This forms the final image that we see and perceive as the external world. The millions of OFFcenter cones project this final image to the brain, inserting it into the visual fields we see as the external image/world.

Contrary to current visual processing paradigm that states images fall onto the retina as an inverted image then reversed in the brain [41], we suggest that the final image we see is not reversed in the brain, but rather inversion never occurs. Instead, images are coiled and compressed graded potentials on the retina that are intensified and amplified in OFF-center photoreceptors and are decompressed and expanded in the brain to the original size of the image, which is perceived by the brain as the external image. The rods and cones see in strips and compress the image onto the discs. The images and their corresponding distances that we see are compressed and coded in reverse on the discs, with the closest part of the image falling on the closest spot on the retina, and the farthest part of the image falling on the back of the retina. The images are coded at depth (not vertically) because of their position in our eyes. The discs on the individual photoreceptors have narrow scope of the visual field [41] suggesting we only see a portion of the image-not the entire image. Only when the rods and cones piece together the larger image on the retina do we see the image in its entirety. This is because the visual fields of the photoreceptors see only a small portion of the actual image, with the strips being similar to millions of tiny eyes with tunnel vision that combine the tiny images into one complete image, with the final image we see is the result of millions of tiny portions of the images being pieced together into a completed, single, magnified image. Similar to pixels on a computer screen or televisions screen, no one pixel or rod/cone sees the whole picture. Additionally, if there is damage to one of the discs, there will be a black spot in the visual field corresponding to that area.

The final external image we perceive results from OFF-center cells projecting the image back to its original location. Clinically speaking, we term this "panoramic retinal processing of the OFFcenter photoreceptors as external visual fields". In order to determine the role of $\mathrm{ON}$-center photoreceptors in visual perception of the final perceived image, more directed studies would be needed.

\section{Conclusion}

A new theory of vision presented in this article explains that retinal cells including the ganglion, horizontal, bipolar, amacrine, and Muller cells, (including photoreceptors) are oscillating at alpha (mu) rhythms and are synchronized with the visual and parietal cortices. This creates and existing "visual space" that forms an infrastructure needed for vision when photoreceptors are stimulated. The cortex sends feedback to the layers in the visual pathway in the retina and lateral geniculate nucleus in the thalamus [70]. This suggests that the brain and retina communicate in real-time without the delay in time it takes to process each electrochemical process to the brain and back to the retina before vision forms. Since the retina is an extension of the brain, and the preexisting oscillatory network has been already set up as embryos, the theory explains how the images formed on the retina are automatically projected into the brain right side up through these fast oscillations. Our theory considers the thalamus as the central hub that integrates all proprioceptive information and projects it into a default space. The retinogeniculo-cortical synchronization results in the final electrical image that we observe from the lateral geniculate nucleus, which we postulate could also be from the fovea, because we observe our world internally from the thalamus.

Furthermore, we have explained that the oscillatory waves play a critical role in afterimages in that oscillations are pre-existing, meaning they originated before we exit the birth canal. After birth, they continue to connect the retina, thalamus, and cortex together allowing the thalamus to perceive retinal images; therefore, we suggest that some cortical involvement is needed to perceive the afterimage. However, the mechanisms underlying both positive and negative afterimages are not well known, so further study of afterimages and visual and retinal processing needs to be done to elucidate these mechanisms and support our proposed model.

\section{Conflicts of interest}

All the authors declare that there were no actual or personal conflicts of interest, including financial, personal, or other relationships, people, or organizations that would influence or be perceived to influence their work while writing this manuscript. All pictures in this article are the original work of the medical artist, and all proposed theories are the original ideas of the authors.

\section{Funding}

All the authors declare that no sponsors, funding, or financial support was granted in the writing of this manuscript.

\section{References}

[1] Jerath R, Crawford MW. Layers of human brain activity: a functional model based on the default mode network and slow oscillations. Front Hum Neurosci 2015;9:248

[2] Utz S, Carbon CC. Afterimages are biased by top-down information. Perception 2015;44(11):1263-74.

[3] Wang $\mathrm{XH}$ et al. Investigating the temporal patterns within and between intrinsic connectivity networks under eyes-open and eyes-closed resting states: a dynamical functional connectivity study based on phase synchronization. PLoS One 2015;10(10):e0140300.

[4] Jerath R, Crawford MW, Barnes VA. Functional representation of vision within the mind: a visual consciousness model based in 3D default space. J Med Hypotheses Ideas 2015;9(1):45-56.

[5] Purves D et al. Neuroscience. 2nd ed. Sunderland, MA: Sinauer Associates; 2001.

[6] Auferkorte $\mathrm{ON}$ et al. $\mathrm{GABA}(\mathrm{A})$ receptors containing the alpha2 subunit are critical for direction-selective inhibition in the retina. PLoS One 2012;7(4): e35109.

[7] Purgert RJ, Lukasiewicz PD. Differential encoding of spatial information among retinal on cone bipolar cells. J Neurophysiol 2015;114(3):1757-72.

[8] Nolte J. The human brain: an introduction to its functional anatomy. St. Louis, MO: Mosby; 1999.

[9] Kramer RH, Davenport CM. Lateral inhibition in the vertebrate retina: the case of the missing neurotransmitter. PLoS Biol 2015;13(12):e1002322.

[10] Walker MT et al. RdgB2 is required for dim-light input into intrinsically photosensitive retinal ganglion cells. Mol Biol Cell 2015;26(20):3671-8.

[11] Hammer M et al. Optical properties of ocular fundus tissues - an in vitro study using the double-integrating-sphere technique and inverse Monte Carlo simulation. Phys Med Biol 1995;40(6):963-78.

[12] Toth CA et al. A comparison of retinal morphology viewed by optical coherence tomography and by light microscopy. Arch Ophthalmol 1997:115(11):1425-8.

[13] Franze K et al. Muller cells are living optical fibers in the vertebrate retina. Proc Natl Acad Sci USA 2007;104(20):8287-92.

[14] Reichenbach A, Robinson SR. Phylogenetic constraints on retinal organisation and development. Prog Retin Eye Res 1995;15(1):139-71.

[15] Nicholls J et al. From neuron to brain. 4th ed. Sinauer Associates Inc.; 2001.

[16] Lucas RJ et al. How rod, cone, and melanopsin photoreceptors come together to enlighten the mammalian circadian clock. Prog Brain Res 2012;199:1-18.

[17] Eysel U. Signal reception and processing by the retina. Ophthalmologe 2007;104(1):79-90. quiz 91.

[18] Malmivuo J, Plonsey R. Bioelectromagnetism: principles and applications of bioelectric and biomagnetic fields. O.U. Press; 1995. Editor.

[19] Baden T et al. Spikes and ribbon synapses in early vision. Trends Neurosci 2013;36(8):480-8. 
[20] tom Dieck S, Brandstatter JH. Ribbon synapses of the retina. Cell Tissue Res 2006;326(2):339-46.

[21] de Souza CF et al. Functional activation of glutamate ionotropic receptors in the human peripheral retina. Exp Eye Res 2012;94(1):71-84.

[22] Tse DY, Chung I, Wu SM. Pharmacological inhibitions of glutamate transporters EAAT1 and EAAT2 compromise glutamate transport in photoreceptor to ON-bipolar cell synapses. Vision Res 2014;103:49-62.

[23] Castilho A et al. Disruption of a neural microcircuit in the rod pathway of the mammalian retina by diabetes mellitus. J Neurosci 2015;35(13):5422-33.

[24] Kaneda M. Signal processing in the mammalian retina. J Nippon Med Sch 2013;80(1):16-24.

[25] Bloomfield SA, Volgyi B. Function and plasticity of homologous coupling between AII amacrine cells. Vision Res 2004;44(28):3297-306.

[26] Watanabe $S$ et al. Prdm13 regulates subtype specification of retinal amacrine interneurons and modulates visual sensitivity. J Neurosci 2015;35 (20):8004-20.

[27] Hu W et al. Ih channels control feedback regulation from amacrine cells to photoreceptors. PLoS Biol 2015;13(4):e1002115.

[28] Chen CK. The vertebrate phototransduction cascade: amplification and termination mechanisms. Rev Physiol Biochem Pharmacol 2005;154:101-21.

[29] Palczewski K. Chemistry and biology of the initial steps in vision: the Friedenwald lecture. Invest Ophthalmol Vis Sci 2014;55(10):6651-72.

[30] Bedggood P, Metha A. Optical imaging of human cone photoreceptors directly following the capture of light. PLoS One 2013;8(11):e79251.

[31] Chen CK. RGS protein regulation of phototransduction. Prog Mol Biol Transl Sci 2015;133:31-45.

[32] Attwell D et al. Signal clipping by the rod output synapse. Nature 1987;328 (6130):522-4

[33] Kramer RH, Molokanova E. Modulation of cyclic-nucleotide-gated channels and regulation of vertebrate phototransduction. J Exp Biol 2001;204(Pt 17):2921-31.

[34] Molday RS, Moritz OL. Photoreceptors at a glance. J Cell Sci 2015;128 (22):4039-45.

[35] Georgiev D. Photons do collapse in the retina not in the brain cortex: evidence from visual illusions. NeuroQuantology 2011;9(2):206-30.

[36] Penn AA, Wong RO, Shatz CJ. Neuronal coupling in the developing mammalian retina. J Neurosci 1994;14(6):3805-15.

[37] Ackman JB, Burbridge TJ, Crair MC. Retinal waves coordinate patterned activity throughout the developing visual system. Nature 2012;490(7419):219-25.

[38] Colonnese MT et al. A conserved switch in sensory processing prepares developing neocortex for vision. Neuron 2010;67(3):480-98.

[39] Kalil RE. The influence of action potentials on the development of the central visual pathway in mammals. J Exp Biol 1990;153:261-76.

[40] Holcman D, Korenbrot JI. The limit of photoreceptor sensitivity: molecular mechanisms of dark noise in retinal cones. J Gen Physiol 2005;125(6):641-60.

[41] Kolb H. How the retina works. Am Sci 2003;91(1):28-35.

[42] Samuel L. On center, off surround ganglion cells. In: Interactive biology. youtube.com.

[43] Balboa RM, Grzywacz NM. The role of early retinal lateral inhibition: more than maximizing luminance information. Vis Neurosci 2000;17(1):77-89.

[44] Wang TM, Holzhausen LC, Kramer RH. Imaging an optogenetic pH sensor reveals that protons mediate lateral inhibition in the retina. Nat Neurosci 2014;17(2):262-8.

[45] Ackman JB, Crair MC. Role of emergent neural activity in visual map development. Curr Opin Neurobiol 2014;24(1):166-75.

[46] Siegel F et al. Peripheral and central inputs shape network dynamics in the developing visual cortex in vivo. Curr Biol 2012;22(3):253-8.
[47] Born RT, Trott AR, Hartmann TS. Cortical magnification plus cortical plasticity equals vision? Vision Res 2015;111(Pt B):161-9.

[48] Castelo-Branco M, Neuenschwander S, Singer W. Synchronization of visual responses between the cortex, lateral geniculate nucleus, and retina in the anesthetized cat. J Neurosci 1998;18(16):6395-410.

[49] Neuenschwander $S$ et al. Feed-forward synchronization: propagation of temporal patterns along the retinothalamocortical pathway. Philos Trans R Soc Lond B Biol Sci 2002;357(1428):1869-76.

[50] Sokoliuk R, VanRullen R. The flickering wheel illusion: when alpha rhythms make a static wheel flicker. J Neurosci 2013;33(33):13498-504.

[51] Bastos AM et al. Simultaneous recordings from the primary visual cortex and lateral geniculate nucleus reveal rhythmic interactions and a cortical source for gamma-band oscillations. J Neurosci 2014;34(22):7639-44.

[52] Klimesch W. Alpha-band oscillations, attention, and controlled access to stored information. Trends Cogn Sci 2012;16(12):606-17.

[53] Katsavos $\mathrm{S}$ et al. Clinical and imaging correlations of generalized hypersynchronous alpha activity in human EEG recordings, during alertness. J Clin Neurophysiol 2015;32(5):413-8.

[54] Ozerdema A et al. Brain oscillations in bipolar disorder in search of new biomarkers. Suppl Clin Neurophysiol 2013;62:207-21.

[55] Nenert R et al. Modulations of ongoing alpha oscillations predict successful short-term visual memory encoding. Front Hum Neurosci 2012;6:127.

[56] Martinovic J, Busch NA. High frequency oscillations as a correlate of visual perception. Int J Psychophysiol 2011;79(1):32-8.

[57] van Ede F et al. Both ongoing alpha and visually induced gamma oscillations show reliable diversity in their across-site phase-relations. J Neurophysiol 2015;113(5):1556-63.

[58] Tzagarakis C, West S, Pellizzer G. Brain oscillatory activity during motor preparation: effect of directional uncertainty on beta, but not alpha, frequency band. Front Neurosci 2015;9:246.

[59] Cruz-Martin A et al. A dedicated circuit links direction-selective retinal ganglion cells to the primary visual cortex. Nature 2014;507(7492):358-61.

[60] Ning T. Development of retinal ganglion cell dendritic structure and synaptic connections. In: Kolb H, Fernandez E, Nelson R, editors. Webvision: the organization of the retina and visual system. Salt Lake City, UT; 1995.

[61] Masland RH. The neuronal organization of the retina. Neuron 2012;76 (2):266-80.

[62] Anstis S, Geier J, Hudak M. Afterimages from unseen stimuli. Iperception 2012;3(8):499-502.

[63] Zaidi Q et al. Neural locus of color afterimages. Curr Biol 2012;22(3):220-4.

[64] Holcombe AO, MacLeod DIA, Mitten ST. Positive afterimages caused by a filledin representation. J Vision 2004;4(8):485.

[65] Shimojo S, Kamitani Y, Nishida S. Afterimage of perceptually filled-in surface. Science 2001;293(5535):1677-80.

[66] Sperandio I, Lak A, Goodale MA. Afterimage size is modulated by size-contrast illusions. J Vis 2012;12(2).

[67] Suffczynski P, Crone NE, Franaszczuk PJ. Afferent inputs to cortical fast-spiking interneurons organize pyramidal cell network oscillations at high-gamma frequencies (60-200 Hz). J Neurophysiol 2014;112(11):3001-11.

[68] Jerath R, Crawford MW. Neural correlates of visuospatial consciousness in 3D default space: insights from contralateral neglect syndrome. Conscious Cogn 2014;28:81-93.

[69] Hirsch JA et al. How inhibitory circuits in the thalamus serve vision. Annu Rev Neurosci 2015;38:309-29.

[70] Ichida JM, Mavity-Hudson JA, Casagrande VA. Distinct patterns of corticogeniculate feedback to different layers of the lateral geniculate nucleus. Eye Brain 2014:2014(6 Suppl. 1):57-73. 